\title{
On Discrete Physics (Digital Philosophy/Digital Cosmology) and the Cellular Automaton: A Perfect Mathematical Deterministic Structure for Reality - as A Huge Computational Simulation
}

\author{
Ramin Zahedi * \\ Logic and Philosophy of Science Research Group, Hokkaido University, Japan
}

Jan 7, 2015

Abstract

In this paper we provide an analysis and overview of some notable definitions, works and thoughts concerning discrete physics (digital philosophy) that mainly suggest a finite and discrete characteristic for the physical world, as well as, of the cellular automaton, which could serve as the basis of a (or the only) perfect mathematical deterministic model for the physical reality.

Keywords: Foundations of Physics, Ontology, Discrete Physics, Discrete Mathematics, Determinism, Reality, Computational Simulation.

“...I consider it quite possible that physics cannot be based on the field concept, i.e., on continuous structures. In that case nothing remains of my entire castle in the air gravitation theory included, -and of- the rest of modern physics." A. Einstein

The concept and etymology of digital is distinct, or "discrete". Digit and its derivatives come from the Latin digitus, meaning finger.

In digital physics (a.k.a. digital philosophy or digital cosmology) it is usually supposed that space, time, body and physical states and quantities are ultimately finite and discrete. Digital philosophy proposes deterministic discrete models for microscopic and fundamental physical processes.

The main reason for this paper is the rising interest of many great contemporary scientists in this field and in particular the recent papers of one of the leading international physicists and Nobel laureate, Prof. Gerard 't Hooft [1-10].

*Email: Zahedi@let.hokudai.ac.jp, Zahedi.R@gmail.com 


\section{Discrete, Finite Physical World}

The physical world has always been described by ordinary calculus and partial differential equations, based on continuous mathematical models. In digital philosophy a different approach is taken, one that often uses the model of cellular automaton (see the next sec.) [15].

Discrete physics (digital philosophy) grew out of an earlier digital physics that proposed to support much of fundamental theories of physics (including quantum theory) in a cellular automaton structure. Specifically, it works through the consequences of assuming that the universe is a gigantic cellular automaton. It is a digital structure that encompasses all of physical reality (including mental activities) as digital processing. From the point of view of determinism, this digital approach to philosophy and physics gets rid of the essentialism of the Copenhagen interpretation of quantum mechanics.

In fact, there is an ongoing effort to understand the physical systems in terms of digital models. According to these models, the universe can be conceived as the output of a universal computer simulation, or as mathematically isomorphic to such a computer, which is a huge cellular automaton [16, 17, 18]. Digital philosophy proposes to find some ways of dealing with certain issues in the philosophy of physics and mind (in particular issues of determinism) [15]. In some sense in this discrete approach to physics, continuity, differentiability, infinitesimals and infinities, are "ambiguous" notions. Despite that, many scientists proposed discrete structures (based on current theories) that can approximate continuous models to any desired degree of accuracy.

Richard Feynman in his famous paper [29], after discussing arguments regarding some of the main physical phenomena concluded that: all these things suggest that it's really true, somehow, that the physical world is representable in a discretized way. It is worth to note here also Einstein's view on continuous models of physics: I consider it quite possible that physics cannot be based on the field concept, i.e., on continuous structures. In that case nothing remains of my entire castle in the air gravitation theory included, -and of- the rest of modern physics [30].

\section{The Cellular Automaton}

Proposals of digital physics reject the very notion of the continuum and claim that current continuous theories are approximations of a true discrete theory of a finite world. Typically such models consist of a regular "lattice" of cells with finite state information at each cell. These lattice cells do not exist in physical space. In fact physical space arises from the relationships between states defined at these cells. In the most commonly studied 
lattice of cells or cellular automaton models, the state is restricted to a fixed number of possibilities.

Firstly, cellular automaton models were studied in the early 1950s. Von Neumann introduced cellular automata more than a half-century ago [21]. By standard definition, a cellular automaton is a collection of stated (or colored) cells on a grid of specified shape that evolves through a number of discrete time steps according to a set of certain rules based on the states of neighboring cells. These rules are then applied iteratively for as many time steps as desired. In fact, von Neumann was one of the first people to consider such a model. The most interesting cellular automaton is something that von Neumann called the universal constructor. The neat thing about cellular automata is that they don't look exactly like computers and there are no such constructs like program, memory or input. They look more like discrete dynamical systems and instead have functionally similar but semantically distinct constructs like evolution rules, space, time and initial conditions.

One of the most fundamental properties of a cellular automaton is a type of grid on which it is calculated or computed. The simplest grid is a one-dimensional line. In two dimensions, square, triangular and hexagonal grids can be considered. Cellular automata can also be built on the Cartesian grids in arbitrary number of dimensions [22, 23]. Cellular automata theory has simple rules and structures that are capable of producing a wide variety of unexpected behaviors. For example, there are universal cellular automata that are able to simulate the behavior of any other cellular automaton [24].

An increasing number of works on cellular automata related to philosophical arguments are being presented by professional scholars interested in the conceptual implications of their work. Among the interesting issues that have already been addressed through the approach of cellular automata in philosophy of science are free will, the nature of computation and simulation, and the ontology of a digital world [25].

\section{Is Discrete Physics a Perfect Deterministic Model for Physical Reality?}

In the opinion of the author, the answer is affirmative [37]. The notion of nature as a discrete form/structure (or a cellular automaton, like a computer simulation model), seems to be supported by an epistemological desideratum and in the last half century many great scientists have logically and reasonably proposed that the physical world might have fundamentally a discrete and computational (or computer simulational) structure [16, 17, $18,20,27,28]$. 
Richard Feynman had speculated that such discrete structures will ultimately provide the most complete and accurate descriptions of physical reality [20]: it always bothers me that, according to the laws as we understand them today, it takes a computing machine an infinite number of logical operations to figure out what goes on in no matter how tiny a region of space, and no matter how tiny a region of time. How can all that be going on in that tiny space? Why should it take an infinite amount of logic to figure out what one tiny piece of space/time is going to do? So I have often made the hypothesis that ultimately physics will not require a mathematical statement, that in the end the machinery will be revealed, and the laws will turn out to be simple, like the chequer board with all its apparent complexities.

As we already noted, Prof. Gerard 't Hooft, a contemporary leading physicist, has also published many papers on this subject in recent years. Particularly, he has tried to consider questions, like:

- Can Quantum Mechanics be Reconciled with Cellular Automata Model?

- Obstacles on the Way Towards the Quantization of Space, Time and Matter -- and Possible Resolutions,

- Does God Play Dice? (One of the Famous Einstein's Ontological Questions),

- The Possibility of a Local Deterministic Theory of Physics,

Here is one of the Gerard 't Hooft's discussions on the possibility of a local deterministic theory of physics [26] (also see [9]): quantum mechanics could well relate to micro-physics the same way thermodynamics relates to molecular physics: it is formally correct, but it may well be possible to devise deterministic laws at the micro scale. Why not? The mathematical nature of quantum mechanics does not forbid this, provided that one carefully eliminates the apparent no-go theorems associated to the Bell inequalities. There are ways to re-define particles and fields such that no blatant contradiction arises. One must assume that all macroscopic phenomena, such as particle positions, momenta, spins, and energies, relate to microscopic variables in the same way thermodynamic concepts such as entropy and temperature relate to local, mechanical variables. The outcome of these considerations is that particles and their properties are not, or not entirely, real in the ontological sense. The only realities in this theory are the things that happen at the Planck scale. The things we call particles are chaotic oscillations of these Planckian quantities.

t'Hooft in his most recent paper [9], (see also [10]), where discussing the mapping between the Bosonic quantum fields and the cellular automaton in two space-time dimensions, concluded that: "the states of the cellular automaton can be used as a basis for 
the description of the quantum field theory. These models are equivalent. This is an astounding result. For generations we have been told by our physics teachers, and we explained to our students, that quantum theories are fundamentally different from classical theories. No-one should dare to compare a simple computer model such as a cellular automaton based on the integers, with a fully quantized field theory. Yet here we find a quantum field system and an automaton that are based on states that neatly correspond to each other, they evolve identically. If we describe some probabilistic distribution of possible automaton states using Hilbert space as a mathematical device, we can use any wave function, certainly also waves in which the particles are entangled, and yet these states evolve exactly the same way. Physically, using 19th century logic, this should have been easy to understand: when quantizing a classical field theory, we get energy packets that are quantized and behave as particles, but exactly the same are generated in a cellular automaton based on the integers; these behave as particles as well. Why shouldn't there be a mapping"?

Of course one can, and should, be skeptic. Our field theory was not only constructed without interactions and without masses, but also the wave function was devised in such a way that it cannot spread, so it should not come as a surprise that no problems are encountered with interference effects, so yes, all we have is a primitive model, not very representative for the real world. Or is this just a beginning"?

He also mentions in his paper concerning three space-time dimensions (for which there is a special interest and emphasis in the literature and relating to the physical reality of three dimensional sub-universe $[11,12,13,14]$ : the classical theory suggests that gravity in three space-time dimensions can be quantized, but something very special happens; ... now that would force us to search for deterministic, classical models for $2+1$ dimensional gravity. In fact, the difficulty of formulating a meaningful 'Schrodinger equation' for a $2+1$ dimensional universe, and the insight that this equation would (probably) have to be deterministic, was one of the first incentives for this author to re-investigate deterministic quantum mechanics as was done in the work reported about here: if we would consider any classical model for $2+1$ dimensional gravity with matter (which certainly can be formulated in a neat way), declaring its classical states to span a Hilbert space in the sense described in our work, then that could become a meaningful, unambiguous quantum system.

In addition, contemporary British physicist, John Barrow states: we now have an image of the universe as a great computer program, whose software consists of the laws of nature which run on hardware composed of the elementary particles of nature [19].

As a special but important case concerning Bell's inequalities, $t$ ' Hooft points out, Bell has shown that hidden variable theories (that the quantum particles are, somehow, accompanied by classical hidden variables that decide what the outcome of any of possible 
measurements will be, even if the measurement is not made) are unrealistic. We must conclude that the cellular automaton theory - the model of $t$ ' Hooft (see $[8,9]$ ) - cannot be of this particular type. Yet, we had a classical system and we claim that it reproduces quantum mechanics with probabilities generated by the squared norm of wave functions. Quantum states, and in particular entangled quantum states, are perfectly legitimate to describe statistical distributions. But to understand why Bell's inequalities can be violated in spite of the fact that we do start off with a classical deterministic, discrete theory (e.g. based on the cellular automaton) requires a more detailed explanation (see [8]). There is also a complete explanation regarding the collapse of the wave function via the cellular automaton structure $[7,8]$.

An immense and relatively newer research field of physics is loop quantum gravity, which may lend support to digital physics, also assumes space-time is "quantized" [32-36].

From the historical perspective it is worth to note that one of the first ideas that "the universe is a computer simulation" was published by Konrad Zuse [16]. He was the first to suggest (in 1967) that the entire universe is being computed on a huge computer, possibly a cellular automaton. In his paper he writes: that at the moment we do not have full digital models of physics ... which would be the consequences of a total discretization of all natural laws? For lack of a complete automata-theoretic description of the universe he continues by studying several simplified models. He discusses neighboring cells that update their values based on surrounding cells, implementing the spread and creation and annihilation of elementary particles. He writes: in all these cases we are dealing with automata types known by the name "cellular automata" in the literature, and cites von Neumann's 1966 book: Theory of self-reproducing automata [16, 31].

\section{Some remarks}

From the above discussions and arguments some logical/ontological questions naturally arise. Are we part of a computer simulation? Are there some advanced civilizations, who have created this huge simulation?, In other words, if we discover that we are existing in a sort of computer simulation, naturally and logically, we can ask, who has created it and is running this simulation, and also for what reason(s)?, Are we a part of a vast scientific and social experiment? Does it made sense to reason that this simulation was created by others?

The ontological structure of a discrete-finite model of reality needs further research. One prospect would be searching for phenomena which cannot be predicted, calculated and described (theoretically/experimentally) according to current quantum theories and other fundamental theories of physics, but could be demystified only by discrete structures [37]. 


\section{References:}

[1]- G. 't Hooft, '"Quantum Mechanics and determinism," in Proceedings of the Eighth Int. Conf. on "Particles, Strings and Cosmology, Univ. of North Carolina, Chapel Hill, Apr. 1015, 2001, P. Frampton and J. Ng, Eds., Rinton Press, Princeton, pp. 275 - 285; ITPUU/01/18, SPIN-2001/11, arXiv:hep-th/0105105; id., Determinism beneath Quantum Mechanics, presented at "Quo vadis Quantum Mechanics?", Temple University, Philadelphia, (September 25, 2002), ITP-UU-02/69, SPIN-2002/45, arXiv:quant$\mathrm{ph} / 0212095,2002$.

[2]- G. 't Hooft, "The mathematical basis for deterministic quantum mechanics, in Beyond the Quantum," World Scientific, Th. M. Nieuwenhuizen et al, ed., pp.3-19, arXiv:quant$\mathrm{ph} / 0604008,2006$

[3]- G. 't Hooft, "Quantum Gravity as a Dissipative Deterministic System," Class. Quant. Grav. 16, 3263, 1999.

[4]- G. 't Hooft, "Determinism in Free Bosons," Int. J. Theor. Phys. 42, 355, 2003.

[5]- G. 't Hooft, "Entangled quantum states in a local deterministic theory," 2nd Vienna Symposium on the Foundations of Modern Physics (June 2009), ITP-UU-09/77, SPIN09/30; arXiv:0908.3408v1 [quant-ph], 2009.

[6]- G. 't Hooft, "Classical cellular Automata and Quantum Field Theory," in Proceedings of the Conference in Honor of Murray Gell-Mann's 80th Birthday "Quantum Mechanics, Elementary Particles, Quantum Cosmology and Complexity", Singapore, February 2010, H. Fritzsch and K. K. Phua, eds., World Scientific, pp 397 - 408, Repr. in: Int. J. Mod. Phys. A25, no 23, pp. 4385-4396, 2010.

[7]- Gerard 't Hooft, "Quantum Mechanics from Classical Logic," Journal of Physics: Conference Series 361, 012024, IOP Publishing, 2012.

[8]- G. 't Hooft, "How a wave function can collapse without violating Schrodinger's equation, and how to understand Born's rule," ITP-UU-11/43, SPIN-11/34, arXiv:1112.1811 [quant-ph], 2011.

[9]- Gerard 't Hooft, "The Cellular Automaton Interpretation of Quantum Mechanics. A View on the Quantum Nature of our Universe, Compulsory or Impossible?," arXiv:1405.1548v2, Jun 2014.

[10]- Gerard 't Hooft, "Duality between a deterministic cellular automaton and a bosonic quantum field theory in 1+1 dimensions," arXiv:1205.4107 [quant-ph], 2012. 
[11]- S. Deser, R. Jackiw and G. 't Hooft, "Three-dimensional Einstein gravity: dynamics of at space," Ann. Phys. 152 p. 220, 1984.

[12]- G. 't Hooft, "Classical N-particle cosmology in 2+1 dimensions," Class. Quantum Grav. 10, S79-S91, 1993.

[13]- G. 't Hooft, "Cosmology in 2+1 dimensions", Nucl. Phys. B30 (Proc. Suppl.) pp. 200-203, 1993.

[14]- G. 't Hooft, "Quantization of point particles in (2+1)-dimensional gravity and spacetime discreteness," Class. Quantum Grav. 13, pp. 1023-1039, arXiv:gr-qc/9601014, 1996.

[15]- E. Fredkin, "An Introduction to Digital Philosophy," International Journal of Theoretical Physics, Vol. 42, No. 2, February 2003.

[16]- Konrad Zuse, "Rechnender Raum," Elektronische Datenverarbeitung, Vol 8., pp. 336-344, 1967.

[17]- K. Zuse, "Calculating Space," Cambridge, MA: MIT Press, 1970. (for some historical details about Zuse's works see also: K. Zuse, "The Computer - My Life," Konrad Zuse (et al.), Springer Verlag, Berlin, 1993).

[18]- K. Zuse, "The Computing Universe," International Journal of Theoretical Physics, 21 (6-7), pp. 589-600, 1982.

[19]- John D. Barrow, "New Theories of Everything," Oxford University Press, 2008.

[20]- Richard Feynman, "The Character of Physical Law," Messenger Lectures, Cornell University, p.57, 1964.

[21]- J. Von Neumann, "The General and Logical Theory of Automata," in Cerebral Mechanisms in Behavior: The Hixon Symposium, New York: John Wiley \& Sons, 1951.

[22]- G. Rao Venkatesh, "Digital Philosophy: Are Cellular Automata Important?," Ribbonfarm Inc. (www.ribbonfarm.com), 2007.

[23]- S. Wolfram, “A New Kind of Science,” Champaign, IL: Wolfram Media, 2002.

[24]- P. Gacs, "Reliable Cellular Automata with Self-Organization." J. Stat. Phys. 103, pp. 45-267, 2001.

[25]- Francesco Berto, Jacopo Tagliabue, "Cellular Automata, \# Cellular Automata as Models of Reality" The Stanford Encyclopedia of Philosophy, 2012.

[26]. Gerard 't Hooft, “Does God Play Dice, Physics World,” December 2005. 
[27]- E. Fredkin, "A New Cosmogony," in Phys. Comp. '92: Proceedings of the Workshop on Physics and Computation, IEEE Computer Society Press, pp. 116-121, 1993.

[28]- S. Wolfram, "Statistical Mechanics of Cellular Automata," Reviews of Modern Physics, 55: 601-644, 1983.

[29]- Richard Feynman, "Simulating Physics with Computers," International Journal of Theoretical Physics, Vol. 21, pp. 467-488, 1982.

[30]- Pierre Speziali, (ed.) "Albert Einstein-Michele Besso: Correspondance 1903-1955," Hermann, Paris, 1972.

[31]- John von Neumann, "The Theory of Self-reproducing Automata," A. Burks (ed.), Univ. of Illinois Press, Urbana, IL, 1966.

[32]- Zizzi, Paola, "A Minimal Model for Quantum Gravity," Mod. Phys. Lett. A20, pp. 645-653, 2005.

[33]- Zizzi, Paola, "Computability at the Planck Scale," arXiv:gr-qc/0412076, 2005.

[34]- A. Marzuoli, M. Rasetti, "Spin Network Quantum Simulator," Phys. Lett. A306, pp. 79-87, 2002.

[35]- A. Marzuoli, M. Rasetti, "Computing Spin Networks," Annals of Physics 318: pp. 345-407, 2005.

[36]- F. Girelli, E. R. Livine, "Reconstructing Quantum Geometry from Quantum Information: Spin Networks as Harmonic Oscillators," Class. Quant. Grav. 22: pp. 32953314, 2005.

[37]- Ramin Zahedi, "On the Logical Structure of the Fundamental Forces of Nature: A New Deterministic Mathematical Approach," Hokkaido University Publs., Japan, January 2015, [A submitted and accepted research project, Ramin (A.) Zahedi, (On "Foundations of Physics"), Japan, 2014 - 2015; And an expanded version of my earlier published articles in the Bulletin of the Lebedev Physics Institute, Russian Academy of Sciences, New York, Springer-Verlag, 1997]; https://www.scribd.com/doc/265058014. 